\title{
Re: Deep microbial analysis of multiple placentas shows no evidence for a placental microbiome. (First comment on BJOG-19-0683.R1 \& BJOG-19-1441.R1)
}

\author{
Judy Cohain ${ }^{1}$ \\ ${ }^{1}$ Hebrew University
}

July 7, 2020

Letter to the Editor, BJOG Exchange

Re: Deep microbial analysis of multiple placentas shows no evidence for a placental microbiome

[Author's title] Logical argument for Placental Microbiome: Learning from Rh negative sensitization

Recent deep microbial analysis of multiple placentas found no evidence for a placental microbiome (1) Donald Dudley (2) says "Yea, No, Maybe. Neither considered what seems to me an obvious argument for likelihood of a placental microbiome. We know in the case of $\mathrm{Rh}$ positive fetuses residing in $\mathrm{Rh}$ negative mothers, that sometimes an adequate amount of fetal red blood cells pass thru placental circulation during the third trimester of pregnancy to sensitize Rh negative mothers, causing them to make antibodies. For that reason we routinely give all $\mathrm{Rh}$ negative mothers who's partners are Rh Positive, a shot of ANTI-D Rhogam at 28 weeks of pregnancy. Red blood cells are 10,000 nm in diameter whereas E coli are $700 \mathrm{~nm}$ in diameter. Bacterial ribosomes are $25 \mathrm{~nm}$ in diameter. Since perhaps tens or hundreds of fetal red blood cells cross the barrier, it seems logical to assume that bacteria, which are less than one tenth the diameter, logically speaking, must also. If there is a flaw in my logic, please do tell! The factors deciding whether or how many bacteria cross over to the fetus are unknown but perhaps they cross over ten times more often than $\mathrm{Rh}$ negative women are sensitized in the third trimester? It would be interesting to know whether these sampled women in the Kuperman et al study and their placentas received the routine prophylactic high dose of IV antibiotics before cesarean surgery causing the cultures to be negative?

Another largely ignored fact is that newborns delivering in hospital, receive a less protective microbiome than those born at home.(3)

Judy Cohain

Hebrew University - Maternal Fetal Medicine

Alon Shvut

Israel

1. Kuperman AA et al. Deep microbial analysis of multiple placentas shows no evidence for a placental microbiome. BJOG 2020; 127: 159-169.

2. Dudley, D. The placental microbiome: yea, nay or maybe? BJOG 2020; 127: 170-170.

3. Combellick JL, Shin H, Shin D, et al. Differences in the fecal microbiota of neonates born at home or in the hospital [published correction appears in Sci Rep. 2019 Jun 18;9(1):9044]. Sci Rep . 2018;8(1):15660. 